\title{
The Efficacy of 2\% Topical Rebamipide on Conjunctival Squamous Metaplasia and Goblet Cell Density in Dry Eye Disease
}

\author{
Cem Simsek, ${ }^{1}$ Murat Dogru, ${ }^{1,2}$ Megumi Shinzawa, ${ }^{1,2}$ Seika Den, ${ }^{2}$ Takashi Kojima, ${ }^{1}$ Hiroyuki Iseda, ${ }^{2}$ Mai Suzuki, ${ }^{2}$ \\ Yoshiyuki Shibasaki, ${ }^{3}$ Naoshi Yoshida, ${ }^{3}$ and Jun Shimazaki ${ }^{2}$
}

\begin{abstract}
Purpose: To clarify the pharmacological effects of $2 \%$ rebamipide eye drops on mucosal membrane functions of the ocular surface epithelium, we investigated keratoconjunctival alterations at the cellular level in this study. Methods: Fifteen patients with definite dry eye disease were recruited from outpatient clinics of the Department of Ophthalmology, Ichikawa General Hospital. The patients received treatment with $2 \%$ rebamipide eye drops q.i.d for 12 weeks. Symptom score assessment, tear film breakup time, fluorescein and lissamine green ocular surface vital staining, grading of lid wiper epitheliopathy, Cochet-Bonnet corneal sensitivity, assessment of squamous metaplasia grades, and goblet cell density calculations from conjunctival impression cytology samples, as well as evaluation of nucleocytoplasmic ratios and corneal epithelial cells from in vivo confocal microscopy images before and 3 months after treatment were performed.

Results: The mean symptom scores, tear film breakup time values, ocular surface fluorescein and lissamine green vital staining scores, and lid wiper scores showed a significant improvement after treatment $(P<0.01)$. The mean squamous metaplasia grade also showed a significant improvement $(1.2 \pm 0.1 \rightarrow 0.3 \pm 0.1) 3$ months after treatment $(P=0.004)$. There were similar significant improvements in the mean corneal epithelial cell density $\left(660.1 \pm 62.6 \rightarrow 1015.5 \pm 43.5\right.$ cells $\left./ \mathrm{mm}^{2}\right)(P=0.002)$ and nucleocytoplasmic ratios $(0.1 \pm 0.0 \rightarrow 0.2 \pm$ $0.0)(P=0.0042)$ after treatment.

Conclusions: Topical use of $2 \%$ rebamipide for 3 months was associated with improvements in ocular surface differentiation due to changes of mucosal functions at the cellular level. These alterations may explain objective and subjective improvements in dry eye disease.
\end{abstract}

Keywords: dry eye syndrome, ocular surface, rebamipide, squamous metaplasia

\section{Introduction}

$\mathbf{T}$ HE DEFINITION OF DRY EYE DISEASE (DED) was updated by the Second International Dry Eye Workshop (DEWS II) Subcommittees in 2017. According to the new consensus report, dry eye is a multifactorial disease of the ocular surface characterized by a loss of homeostasis of the tear film and accompanied by ocular symptoms, in which tear film instability and hyperosmolarity, ocular surface inflam- mation and damage, and neurosensory abnormalities play etiological roles. ${ }^{1}$

The new DEWS report suggests a staged treatment of dry eye disease based on severity with a shift in treatment algorithm from simple lubrication to addition of mucin and water secretagogues. Among such agents, rebamipide is a novel agent that can increase mucin secretion from the keratoconjunctival epithelium, increase the conjunctival goblet cell density, and decrease ocular surface epithelial

${ }^{1}$ Department of Ophthalmology, Keio University School of Medicine, Tokyo, Japan.

${ }^{2}$ Department of Ophthalmology, Tokyo Dental College, Ichikawa General Hospital, Chiba, Japan.

${ }^{3}$ Department of Medical Affairs, Otsuka Pharmaceutical Co., Ltd., Tokyo, Japan.

(C) Cem Simsek et al. 2019; Published by Mary Ann Liebert, Inc. This Open Access article is distributed under the terms of the Creative Commons Attribution Noncommercial License (http://creativecommons.org/licenses/by-nc/4.0/) which permits any noncommercial use, distribution, and reproduction in any medium, provided the original author(s) and the source are cited. 
damage scores. ${ }^{2-6}$ Initial clinical studies disclosed the safety of rebamipide together with its favorable effects on symptoms and vital staining scores. ${ }^{7,8}$ Long-term studies reconfirmed these facts leading to approval of $2 \%$ rebamipide as a mucin secretagogue in the treatment of dry eyes in Japan in September 2011. Rebamipide elevates expression levels of MUC1, MUC4, and MUC16 through activation of the epidermal growth factor receptor and increases mucin-like glycoprotein production in human corneal epithelial cells. ${ }^{4,6}$ Experimental studies after 2011 disclosed effects related to improvement of the ocular surface ultrastructural environment, increased secretion of membrane-bound mucins, and improvement of the epithelial barrier. $^{9-11}$

Squamous metaplasia of the keratoconjunctival epithelium, loss of goblet cells, and lid wiper epitheliopathy (LWE) have been demonstrated to be associated with DED. ${ }^{12-16} \mathrm{Ad}-$ ditionally, squamous metaplasia resulting from disturbances in healthy epithelial differentiation has been already reported in dry eye patients. ${ }^{14}$ This study aimed to investigate keratoconjunctival alterations at the cellular level and pharmacological effects with 3 months of $2 \%$ rebamipide eye drop treatment on mucosal membrane functions of the ocular surface epithelium in dry eye patients.

\section{Methods}

\section{Patients}

Fifteen dry eye patients were recruited at Tokyo Dental College, Ichikawa General Hospital, during June 2016 and March 2017 (1 male, 14 females; mean age: 64.9 years). None of the patients had a history of Stevens-Johnson syndrome, chemical, thermal, or radiation injury, or any other systemic/ocular disorder; underwent any ocular surgery; or had contact lens use that would create an ocular surface problem. The details of patient recruitment are shown in Fig. 1. The patients received rebamipide eye drops (Mucosta ${ }^{\circledR}$ Ophthalmic Suspension UD 2\%; Otsuka Pharmaceutical Co., Ltd., Tokyo) q.i.d for 12 weeks and underwent the examinations shown in Supplementary Fig. S1. The study was approved by the institutional review board of Ichikawa General Hospital, Chiba (Approval No. I 15-56), and adhered to tenets of the Declaration of Helsinki. Written informed consent was received from all patients

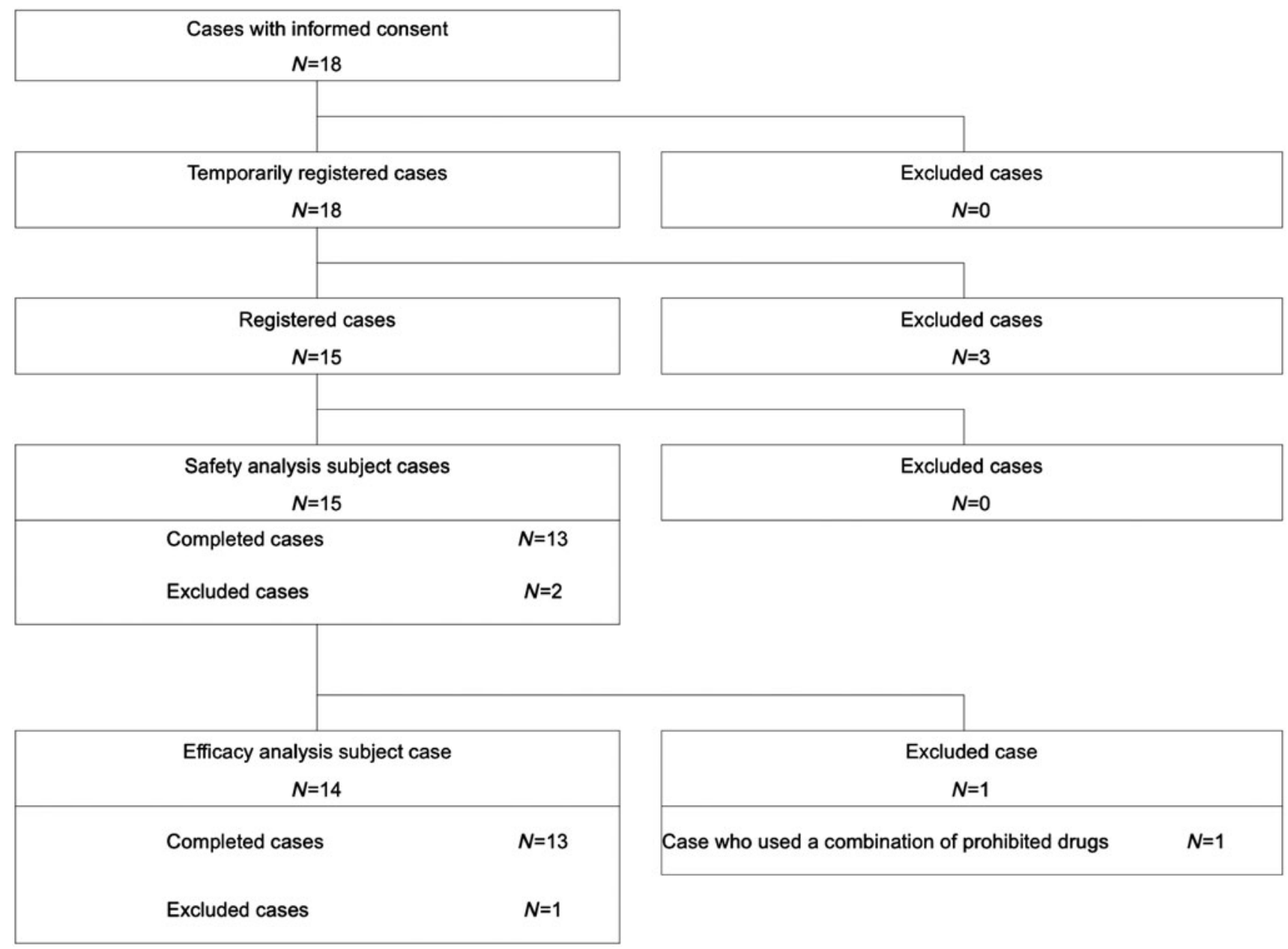

FIG. 1. Patient recruitment details. Patients who are over 20 years old (legal age for acquiring an informed consent in Japan) with 2 or more severe dry eye-related symptoms (foreign body sensation, dryness, photophobia, eye pain, and blurred vision), fluorescein corneal staining score of 3 or more, lissamine green conjunctival staining score of 4 or more, and Schirmer test (5-min value) value of $5 \mathrm{~mm}$ or less were included in this study. 
after informational material about examination procedures was provided to them. All dry eye examinations were performed from noninvasive to more invasive tests in the order reported in the Methods section according to guidelines of the Second International DEWS report. ${ }^{1}$ The examiners were not allowed to see the results of previous examinations of the patients, which was monitored by the investigation coordinator. The examiners were blinded to patient names and dates of examinations when they were evaluating the impression cytology specimens and confocal microscopy images.

\section{Dry eye-related ocular symptom score assessment}

A validated ${ }^{17,18} 5$-item questionnaire of ocular symptoms relating to dry eyes was used to assess the severity of dry eye disease, which included the following questions: (1) Do you have any foreign body sensation in your eye? (2) Do you feel dryness in your eye? (3) Have your eyes ever had a dazzle sensation? (4) Do you feel pain in your eye? (5) Do you have blurred vision? The severity of dry eye symptoms, including foreign body sensation, dryness, dazzle, pain, and blurred vision, was graded as follows: 0: no symptoms, 1: mild, 2: moderate, 3: severe, and 4: extremely severe. The worst symptom was recorded for each patient. Symptoms of ocular discomfort were evaluated during scheduled visits by self-administered queries. Only symptom data from patient diaries (using the entries immediately before each scheduled visit) are presented.

\section{Tear film breakup time measurements}

The standard tear film breakup time measurement was performed. Two microliters of $1 \%$ fluorescein dye was instilled into the conjunctival sac as previously reported. ${ }^{19}$ The interval between the last complete blink and the appearance of the first corneal black spot in the stained tear film was measured 3 times and the mean value of the measurements was calculated.

\section{Fluorescein and lissamine green ocular surface vital staining}

BUT measurement was followed by staining with $1 \%$ lissamine green solution. Fluorescein and lissamine green staining of the ocular surface was noted and scored. Fluorescein and lissamine green staining scores followed the National Eye Institute/Industry Workshop guidelines; corneas were divided into 5 and conjunctivae into 6 zones with a staining score between 0 and 3 points in each zone (Supplementary Fig. S2).

\section{Grading of LWE}

Yamamoto et al. reported a new grading of LWE by modifying the grade scale of Korb et al. ${ }^{15,16}$ According to the new grading scale, the horizontal length and sagittal width of the lissamine green staining were determined in each eyelid. The grading of LWE was done by the same ophthalmologist carrying out a careful examination to differentiate the thin line of vital dye staining in the mucocutaneous junction denoting Marx's line. ${ }^{20,21}$

\section{Assessment of squamous metaplasia grades}

The impression cytology specimens were obtained after administration of topical anesthesia with $0.4 \%$ oxybupro- caine hydrochloride and tetracaine hydrochloride (Alcon Cusi S.A., Barcelona, Spain). Strips of cellulose acetate filter paper (HAWP 01300; Millipore, Bedford, MA) that were soaked in distilled water for a few hours and dried at room temperature were applied on the nasal bulbar conjunctiva adjacent to the corneal limbus, pressed gently by a forceps, and then removed. The specimens were then fixed with $10 \%$ formaldehyde, stained with periodic acid-Schiff, dehydrated in ascending grades of ethanol and then with xylene, and finally cover-slipped. Quantitative studies of conjunctival epithelial cells were conducted by taking photographs with a calibrated grid under a light microscope at a magnification of $200 \times$. We photographed 10 different areas of each sample selected at random. We calculated the mean individual epithelial cell area (MIECA) and nucleocytoplasmic (N/C) ratio using ImageJ software (National Institutes of Health, Bethesda, MD) and averaged the outcomes.

\section{Goblet cell density calculations}

Grading of squamous metaplasia was performed according to Tseng grading. ${ }^{22}$ In this grading system, squamous metaplasia is based on cell size, N/C ratio, and goblet cell densities. $^{22,23}$ Goblet cell density calculations from conjunctival impression cytology samples as well as evaluation of N/C ratios and corneal epithelial cells from in vivo confocal microscopy images before and 3 months after treatment were also performed.

\section{In vivo laser scanning confocal microscopy}

We performed ocular surface examinations by in vivo laser scanning confocal microscopy. The Rostock Cornea Module of the Heidelberg Retinal Tomograph II (HRT) (Heidelberg Engineering, Heidelberg, Germany) was used to examine corneal alterations. The examination of patients was performed according to our previous protocol. ${ }^{24}$ Briefly, corneal epithelial cell density and N/C ratios were calculated.

\section{Statistical analyses}

Data are expressed as mean \pm SE. The Wilcoxon signedrank test was performed to compare the examination values before and after rebamipide ophthalmic solution treatment. For all statistical analyses, Prism 6 (Graph-pad, CA) was used. A $P$ value less than $5 \%$ was considered to be statistically significant.

\section{Results}

\section{Assessment of symptom scores}

Compared with baseline scores, the mean score change for foreign body sensation, ocular dryness, photophobia, and worst symptom was $1.9 \pm 0.8,2.3 \pm 0.9,1.9 \pm 1.0$, and $2.6 \pm 0.8$ points, respectively. There was a significant improvement in foreign body sensation, ocular dryness, photophobia, and worst symptoms after $2 \%$ rebamipide treatment at each visit $(P<0.01)$.

At baseline, the mean score for blurred vision ranged from 1.4 to 2.9 (mild to moderate) in 13 patients. Significant improvements from baseline in blurred vision were seen after 12 weeks of $2 \%$ rebamipide eye drop application. The magnitude of improvement in blurred vision was $2.3 \pm 1.3$ 
after 12 weeks of $2 \%$ rebamipide eye drop application $(P<0.05)$.

Compared with baseline scores, the mean score change for eye pain was $1.4 \pm 0.5$ points after 12 weeks of $2 \%$ rebamipide eye drop application $(P>0.05)$. However, the mean eye pain score showed no statistically significant differences among patients after 12 weeks of $2 \%$ rebamipide eye drop application (Fig. 2).

\section{Assessment of tear film breakup times}

The mean of the change in tear film breakup time score was $1.9 \mathrm{~s}$ at 4 weeks, $3.1 \mathrm{~s}$ at 8 weeks, and $4.1 \mathrm{~s}$ at 12 weeks, showing a significant and consistent increase $(P<0.01)$.

\section{Assessment of vital staining scores}

The mean of the change in fluorescein corneal staining score was -2.1 points at 4 weeks, -2.3 points at 8 weeks, and -2.7 points at 12 weeks, showing a significant and consistent decrease during the follow-up period $(P<0.01)$. Likewise, the mean of the change in lissamine green corneal staining score was -3.4 points at 4 weeks, -4.0 points at 8 weeks, and -4.1 points at 12 weeks, showing a significant and consistent decrease $(P<0.01)$ (Fig. 3).

\section{Changes in lid wiper scores}

The mean of the change in lid wiper score significantly was -0.2 points at 4 weeks, -0.3 points at 8 weeks, and -0.5 points at 12 weeks, showing a significant and consistent decrease $(P<0.05)$ (Fig. 4$)$.

\section{Assessment of squamous metaplasia grades and goblet cell densities}

The mean squamous metaplasia grade was $1.2 \pm 0.1$ at baseline, $1.0 \pm 0.1$ at 4 weeks, and $0.3 \pm 0.1$ at 12 weeks. The mean change in squamous metaplasia grade was significant at 4 weeks $(-0.2)$ and at 12 weeks $(-0.8)$ after initiation of treatment $(P<0.01)$. The goblet cell density was $8.3 \pm 14.2$ cells $/ \mathrm{mm}^{2}$ at baseline, $5.7 \pm 5.5$ cells $/ \mathrm{mm}^{2}$ at 4 weeks, and $24.9 \pm 18.4$ cells $/ \mathrm{mm}^{2}$ at 12 weeks. The mean change in goblet cell density increased significantly by $13.3 \mathrm{cells} / \mathrm{mm}^{2}$ at 12 weeks after initiation of $2 \%$ rebamipide treatment $(P<0.01)$ (Fig. 5).

\section{Assessment of in vivo confocal microscopy findings}

The mean of the corneal epithelial cell density increased from $660.1 \pm 62.6$ to $1015.5 \pm 43.5$ cells $/ \mathrm{mm}^{2}$ after 12 weeks of rebamipide treatment. The mean of the corneal epithelial cell density change showed a significant increase of 355.4 cells 12 weeks after initiation of treatment $(P<0.01)$ (Fig. 6). The mean of the N/C ratio was $0.1 \pm 0.0$ at baseline and $0.2 \pm 0.0$ at 12 weeks. The mean change in N/C ratio also showed a significant increase of 0.1 at 12 weeks after treatment $(P<0.01)$ (Fig. 6).

\section{Conclusions}

It has been reported that topical administration of rebamipide significantly improves symptoms, corneal fluorescein staining, conjunctival lissamine green staining, and tear film breakup time in DED. ${ }^{7,10,17}$ Previous animal studies have shown that rebamipide instillation also improved ocular surface epithelial differentiation with a

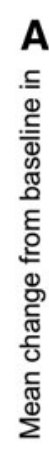

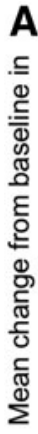

A

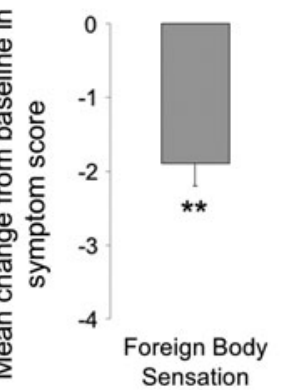

$(\mathrm{N}=9)$

B
Change of Dry eye related symptom at 12 weeks
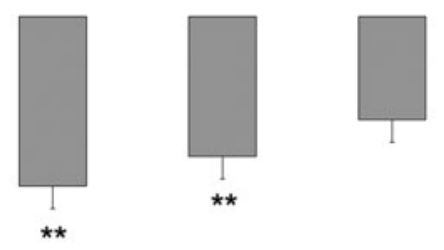

Mean \pm S.E

Dryness

Photophobia

Eye Pain

Blurred Vision Worst symptom

$(\mathrm{N}=5) \quad(\mathrm{N}=8)$

$(\mathrm{N}=13)$

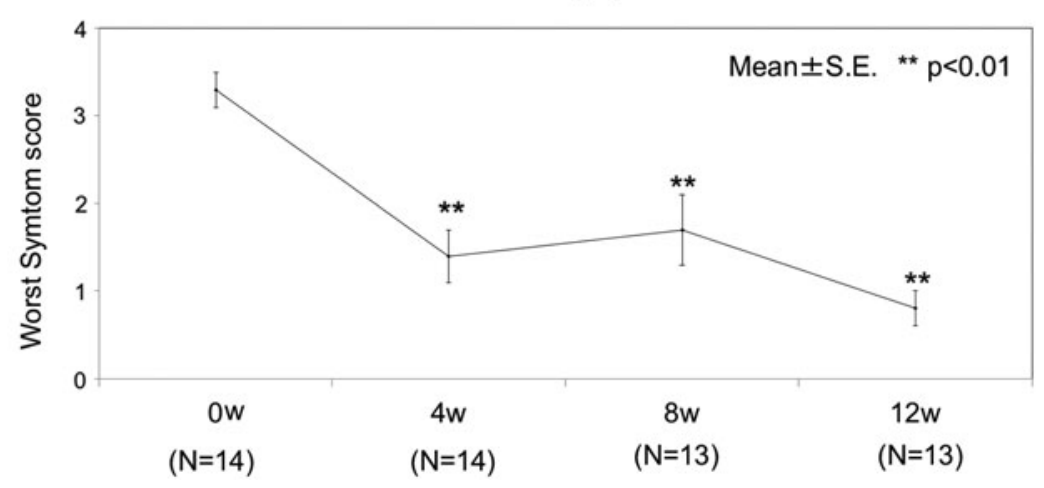

FIG. 2. Symptom score alteration graphs. There was a significant improvement in foreign body sensation, ocular dryness, photophobia, and worst symptoms after $2 \%$ rebamipide treatment at several visits. *Represents $P<0.05$, ** Represents $P<0.01$. 

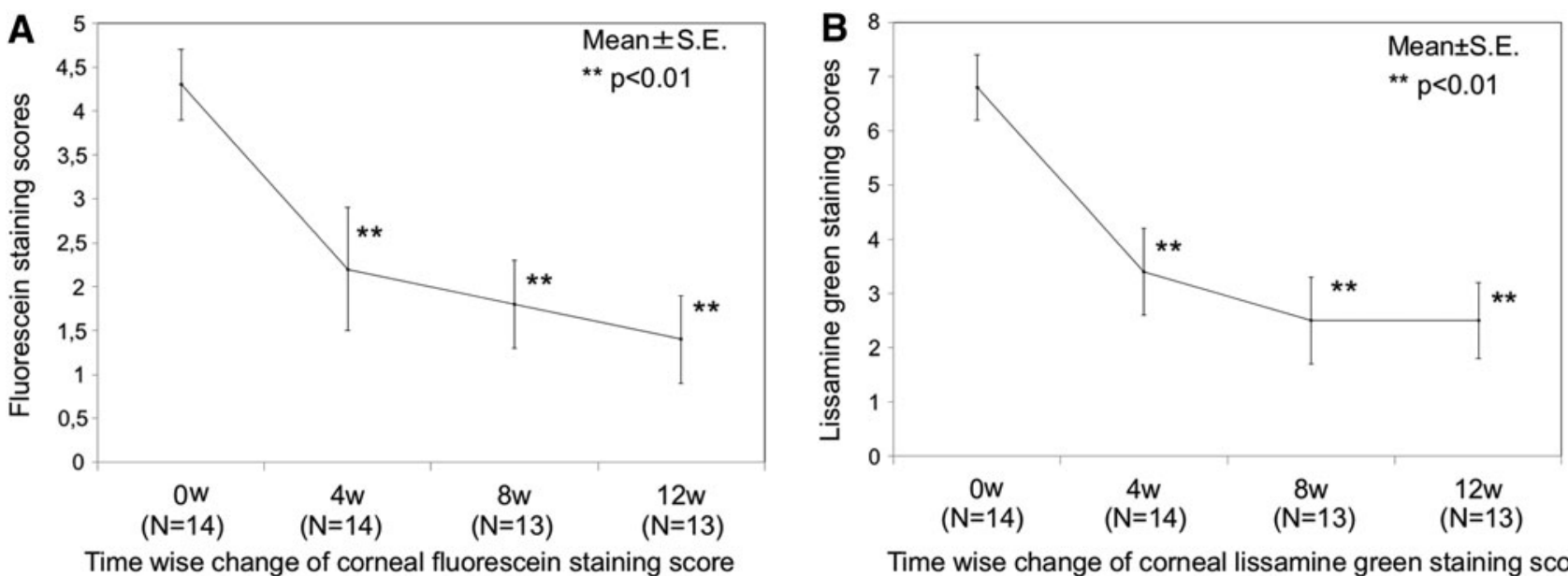

FIG. 3. Alterations of corneal fluorescein and conjunctival lissamine green staining scores after 12 weeks of $2 \%$ rebamipide instillation. A significant and consistent decrease can be seen in the mean corneal fluorescein and conjunctival lissamine green corneal staining score after 12 weeks of $2 \%$ rebamipide treatment in graphs. $* *$ Represents $P<0.01$.

reduction in keratinization. ${ }^{10}$ Additionally, Kinoshita et al. have shown that even short-term rebamipide instillation has favorable therapeutic effects on corneal staining scores. Moreover, they also have demonstrated that administration of rebamipide ophthalmic solution results in significant improvement in both the objective signs and subjective symptoms of DED. ${ }^{18}$ Consistent with these previous studies, we also demonstrated that dry eye-related ocular symptoms, tear break up time (TBUT), corneal, and conjunctival vital staining scores showed a significant improvement after 12 weeks of $2 \%$ rebamipide eye drop administration in the current clinical research.

Previous studies reported that blinking and especially upper eyelid movements play a key role in the dissemination of tear film and maintenance of integrity and homeostasis of the ocular surface. ${ }^{25,26}$ LWE was first defined in detail by
Korb et al., which causes dry eye symptoms with bandshaped corneal erosions that can be diagnosed with a mixture of fluorescein and lissamine green ocular staining at the upper eyelid. ${ }^{27}$ It has been shown that LWE was associated with inflammation of the ocular surface and inflammation can occur due to mechanical forces during blinking. ${ }^{28} \mathrm{Re}$ bamipide is known to decrease the levels of TNF- $\alpha$ and IL- 8 in gastric mucosa as well as the concentration of proinflammatory cytokines in serum. ${ }^{29}$ In human umbilical vein endothelial cells, rebamipide inhibits TNF- $\alpha$-induced IL- 8 expression by suppressing the NF- $\kappa \mathrm{B}$ signaling pathway. ${ }^{30}$ Recently, Ogawa et al. ${ }^{31}$ demonstrated that rebamipide appeared to suppress inflammation by decreasing IL-6 levels in mouse conjunctival epithelial cell cultures. Itakura et al. reported that administration of rebamipide eye drops treats the LWE by an anti-inflammatory effect ${ }^{9}$ and this result
FIG. 4. Timewise change of lid wiper score. The mean of the change in lid wiper score showed a significant and consistent decrease after 12 weeks of $2 \%$ rebamipide treatment in graphs $(P<0.05)$.

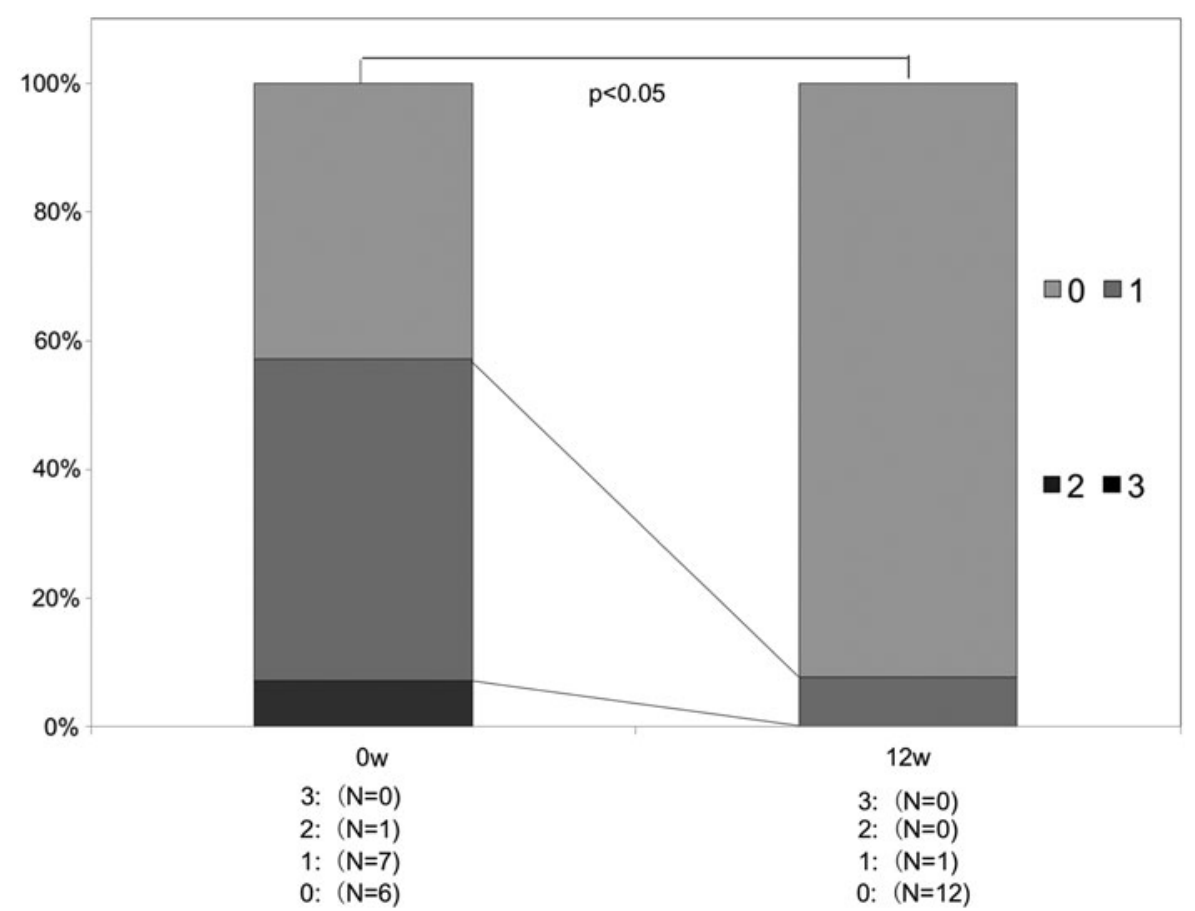



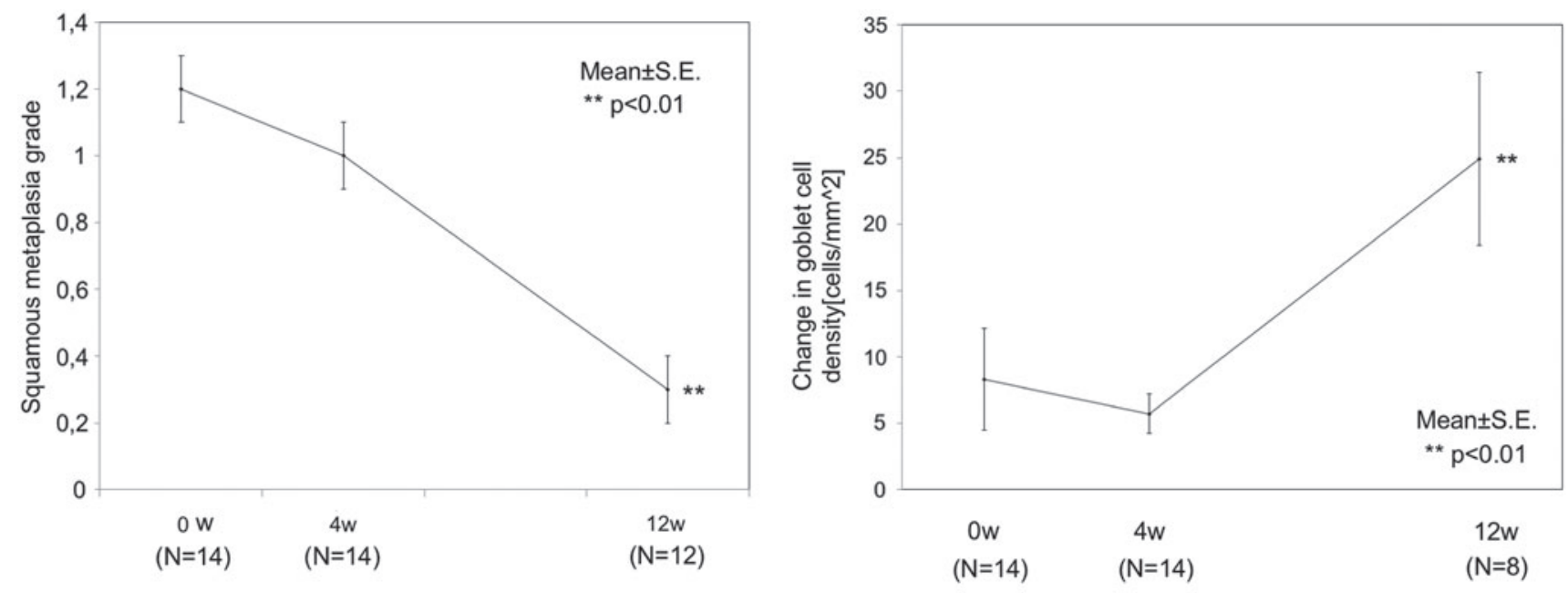

Representative conjunctival IC findings
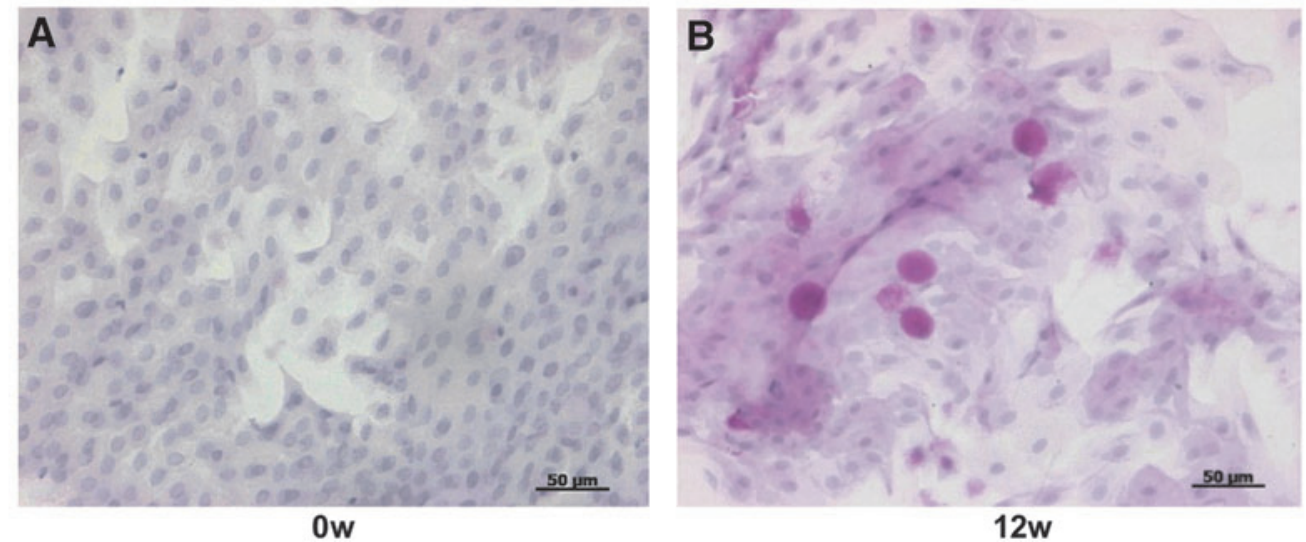

FIG. 5. Conjunctival impression cytology findings and representative conjunctival IC images. The mean change in squamous metaplasia grade decreased and the mean change in GC density increased significantly at 4 and 12 weeks after treatment with $2 \%$ rebamipide (**represents $P<0.01$ ). (A) Note the squamous metaplasia and absence of goblet cells at first visit. (B) Note the improvement of squamous metaplasia and increase of goblet cells after 12 week of $2 \%$ rebamipide treatment. GC, goblet cell; IC, impression cytology.

from previous research is consistent with the current study in terms of the improvement effect of rebamipide in LWE.

On the ocular surface, squamous metaplasia is generally observed following long-term tear film disturbance ${ }^{32}$ and has been reported in a variety of ocular diseases, including Stevens-Johnson syndrome, chronic dry eye, ocular cicatricial pemphigoid, and chemical burns. ${ }^{33-35}$ Lack of lacrimal secretion may also induce the pathological process of squamous metaplasia in the eye. ${ }^{32}$ Squamous metaplasia and limbal stem cell deficiency are known as the 2 primary types of ocular surface dysfunctions, which can cause visual loss and blindness as well. Recent studies have shown that there is a strong relationship between pathologic squamous metaplasia and certain types of lymphocyte/cytokine-mediated chronic inflammations of the ocular surface ${ }^{36,37}$ In the treatment of ocular surface squamous metaplasia, amniotic membrane patching, anti-inflammatory drugs, retinoid therapy, and autologous serum administration are commonly used for its management. In addition to these treatment options, there is still a need to develop new effective treatment modalities in management of squamous meta- plasia on the ocular surface. According to our clinical study results, the mean change in conjunctival squamous metaplasia grade decreased significantly after 12 weeks of $2 \%$ rebamipide treatment. We demonstrated that $2 \%$ rebamipide eye drops have a notable improving effect in conjunctival squamous metaplasia on the ocular surface.

Sufficient amounts of goblet cells play an essential role in maintaining a healthy ocular surface and conjunctival differentiation. ${ }^{38}$ It was recently shown that several types of ocular surface diseases such as keratoconjunctivitis sicca, Sjögren syndrome, and ocular cicatricial pemphigoid were associated with goblet cell loss. ${ }^{39,40}$ Previous human and animal studies demonstrated that rebamipide increases the density of periodic acid-Schiff-positive goblet cells and mucin levels in the cornea and conjunctival epithelial cells. ${ }^{2,6,10,41}$ Additionally, Ohguchi et al. demonstrated that $2 \%$ rebamipide application increased the goblet cell density as well as Muc5AC mRNA expression in an SOD1 knockout mouse model. ${ }^{5}$ The mean of the goblet cell density along with corneal epithelial cell density showed a notable increase with $2 \%$ rebamipide application in this study and this result was consistent with findings of previous studies 

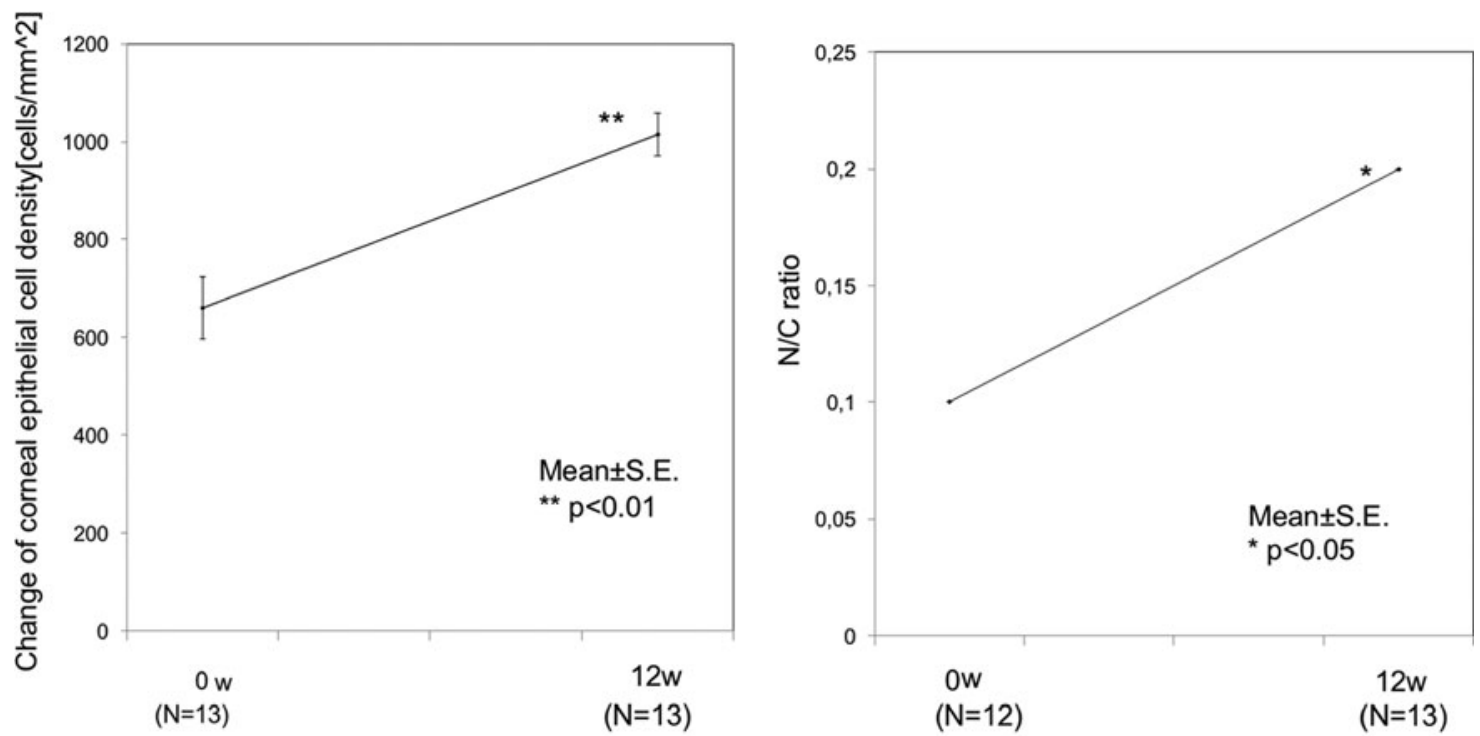

Representative corneal confocal microscopy findings

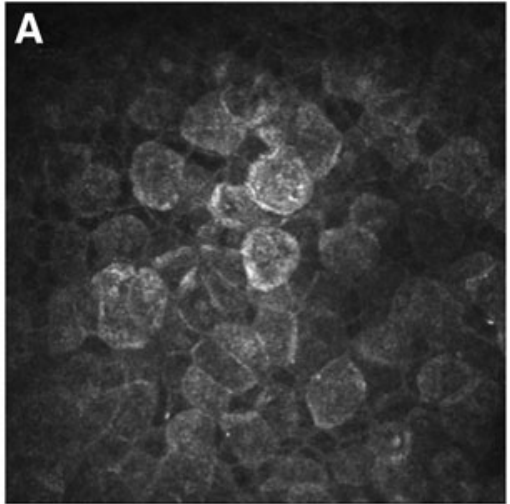

Ow

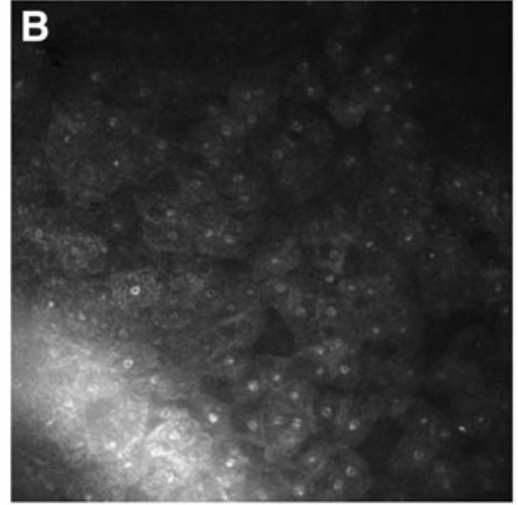

$12 w$

FIG. 6. Confocal microscopy corneal findings and representative corneal confocal microscopy images. The mean of corneal epithelial cells and the N/C ratio density increased significantly after 12 weeks of $2 \%$ rebamipide treatment (**represents $P<0.01$ ). (A) Note the squamous metaplasia of the corneal epithelium, weakening of intercellular cohesion, and decrease of superficial cells at first visit. (B) Note the improvement of pretreatment findings with increase of superficial cells. N/C, nucleocytoplasmic.

suggesting favorable effects on epithelial proliferation an$\mathrm{d} /$ or differentiation.

Our study is the initial investigation looking into cytological changes at the ocular surface by employing impression cytology and in vivo confocal microscopy. Based on improvements of conjunctival squamous metaplasia (Tseng grading), corneal epithelial cell density, and N/C ratios, the combination of $2 \%$ rebamipide eye drops with nonpreserved artificial tears (Soft Santear; Santen Pharmaceutical Co., Ltd., Osaka, Japan) has been thought to have favorable effects on epithelial differentiation, which in turn resulted in concurrent improvements in dry eye symptom scores and tear stability. Future studies with a higher number of patients would definitely strengthen the conclusions of the current study.

\section{Acknowledgments}

This study was supported by a grant from Otsuka Pharmaceuticals to Tokyo Dental College, Ichikawa Gen- eral Hospital. Otsuka was not involved in any part of the conduct of the study, nor were the investigators paid employees of Otsuka. M.D. has received research support or speakers' honoraria from, or has served as a consultant to, Otsuka Pharmaceutical Co., Ltd., Alcon Japan Ltd., Kobayashi Pharmaceutical Co,. Ltd., and Nihon Eco Denki. T.K. has received research support or speakers' honoraria from, or has served as a consultant to, Otsuka Pharmaceutical Co., Ltd., STAAR Japan, Inc., Santen Pharmaceutical, Co., Ltd., and Johnson \& Johnson K.K. J.S. has received research support or speakers' honoraria from, or has served as a consultant to, Otsuka Pharmaceutical Co., Ltd., Santen Pharmaceutical Co., Ltd., Senju Pharmaceutical Co., Ltd., Alcon Japan Ltd., Eye Lens Ltd., Hoya Co., Ltd., Abbott Medical Optics, and Kowa Co., Ltd. Y.S. and N.Y. are employees of Otsuka Pharmaceutical Co., Ltd. This work was presented at the 2018 ARVO annual meeting on Apr 29-May 3, 2018, Honolulu, Hawaii, USA. M.D. and C.S. request acknowledgment of double first authorship. 


\section{Author Disclosure Statement}

No competing financial interests exist.

\section{Supplementary Material}

Supplementary Figure S1

Supplementary Figure S2

\section{References}

1. Craig, J.P., Nichols, K.K., Akpek, E.K., et al. TFOS DEWS II Definition and Classification Report. Ocul Surf 15:276283, 2017.

2. Urashima, H., Takeji, Y., Okamoto, T., Fujisawa, S., and Shinohara, H. Rebamipide increases mucin-like substance contents and periodic acid Schiff reagent-positive cells density in normal rabbits. J Ocul Pharmacol Ther 28:264270, 2012.

3. Urashima, H., Okamoto, T., Takeji, Y., Shinohara, H., and Fujisawa, S. Rebamipide increases the amount of mucinlike substances on the conjunctiva and cornea in the $\mathrm{N}$ acetylcysteine-treated in vivo model. Cornea 23:613-619, 2004.

4. Itoh, S., Itoh, K., and Shinohara, H. Regulation of human corneal epithelial mucins by rebamipide. Curr Eye Res 39: 133-141, 2014.

5. Ohguchi, T., Kojima, T., Ibrahim, O.M., et al. The effects of $2 \%$ rebamipide ophthalmic solution on the tear functions and ocular surface of the superoxide dismutase-1 (sod1) knockout mice. Invest Ophthalmol Vis Sci 54:7793-7802, 2013.

6. Takeji, Y., Urashima, H., Aoki, A., and Shinohara, H. Rebamipide increases the mucin-like glycoprotein production in corneal epithelial cells. J Ocul Pharmacol Ther 28: 259-263, 2012.

7. Kinoshita, S., Awamura, S., Nakamichi, N., et al. A multicenter, open-label, 52-week study of $2 \%$ rebamipide (OPC12759) ophthalmic suspension in patients with dry eye. Am J Ophthalmol 157:576-583.e571, 2014.

8. Dogru, M., Nakamura, M., Shimazaki, J., and Tsubota, K. Changing trends in the treatment of dry-eye disease. Expert Opin Investig Drugs 22:1581-1601, 2013.

9. Itakura, H., Kashima, T., Itakura, M., Akiyama, H., and Kishi, S. Topical rebamipide improves lid wiper epitheliopathy. Clin Ophthalmol 7:2137-2141, 2013.

10. Kojima, T., Simsek, C., Igarashi, A., et al. The role of $2 \%$ rebamipide eye drops related to conjunctival differentiation in superoxide dismutase-1 (Sod1) knockout mice. Invest Ophthalmol Vis Sci 59:1675-1681, 2018.

11. Arakawa, T., Higuchi, K., Fujiwara, Y., et al. 15th anniversary of rebamipide: looking ahead to the new mechanisms and new applications. Dig Dis Sci 50 Suppl 1:S3-S11, 2005.

12. Nelson, J.D., and Wright, J.C. Conjunctival goblet cell densities in ocular surface disease. Arch Ophthalmol 102: 1049-1051, 1984.

13. Tseng, S.C., Hirst, L.W., Maumenee, A.E., Kenyon, K.R., Sun, T.T., and Green, W.R. Possible mechanisms for the loss of goblet cells in mucin-deficient disorders. Ophthalmology 91:545-552, 1984.

14. De Paiva, C.S., Villarreal, A.L., Corrales, R.M., et al. Dry eye-induced conjunctival epithelial squamous metaplasia is modulated by interferon- $\gamma$. Invest Ophthalmol Vis Sci 48: 2553-2560, 2007.

15. Korb, D.R., Herman, J.P., Blackie, C.A., et al. Prevalence of lid wiper epitheliopathy in subjects with dry eye signs and symptoms. Cornea 29:377-383, 2010.
16. Yamamoto, Y., Shiraishi, A., Sakane, Y., Ohta, K., Yamaguchi, M., and Ohashi, Y. Involvement of eyelid pressure in lid-wiper epitheliopathy. Curr Eye Res 41:171-178, 2016.

17. Kinoshita, S., Awamura, S., Oshiden, K., et al. Rebamipide (OPC-12759) in the treatment of dry eye: a randomized, double-masked, multicenter, placebo-controlled phase II study. Ophthalmology 119:2471-2478, 2012.

18. Kinoshita, S., Oshiden, K., Awamura, S., et al. A randomized, multicenter phase 3 study comparing $2 \%$ rebamipide (OPC-12759) with $0.1 \%$ sodium hyaluronate in the treatment of dry eye. Ophthalmology 120:1158-1165, 2013.

19. Kaido, M., Ishida, R., Dogru, M., Tamaoki, T., and Tsubota, K. Efficacy of punctum plug treatment in short breakup time dry eye. Optom Vis Sci 85:758-763, 2008.

20. Donald, C., Hamilton, L., and Doughty, M. A quantitative assessment of the location and width of Marx's line along the marginal zone of the human eyelid. Optom Vis Sci 80: 564-572, 2003.

21. Norn, M. Meibomian orifices and Marx's line. Studied by triple vital staining. Acta Ophthalmol (Copenh) 63:698700, 1985.

22. Tseng, S.C. Staging of conjunctival squamous metaplasia by impression cytology. Ophthalmology 92:728-733, 1985.

23. Nelson, J.D. Impression cytology. Cornea 7:71-81, 1988.

24. Kojima, T., Matsumoto, Y., Dogru, M., and Tsubota, K. The application of in vivo laser scanning confocal microscopy as a tool of conjunctival in vivo cytology in the diagnosis of dry eye ocular surface disease. Mol Vis 16: 2457, 2010.

25. Goto, E., and Tseng, S.C. Kinetic analysis of tear interference images in aqueous tear deficiency dry eye before and after punctal occlusion. Invest Ophthalmol Vis Sci 44: 1897-1905, 2003.

26. Bron, A.J., Tiffany, J.M., Gouveia, S.M., Yokoi, N., and Voon, L.W. Functional aspects of the tear film lipid layer. Exp Eye Res 78:347-360, 2004.

27. Korb, D.R., Greiner, J.V., Herman, J.P., et al. Lid-wiper epitheliopathy and dry-eye symptoms in contact lens wearers1. Eye Contact Lens 28:211-216, 2002.

28. Berry, M., Pult, H., Purslow, C., and Murphy, P.J. Mucins and ocular signs in symptomatic and asymptomatic contact lens wear. Optom Vis Sci 85:E930-E938, 2008.

29. Fujioka, T., Arakawa, T., Shimoyama, T., et al. Effects of rebamipide, a gastro-protective drug on the Helicobacter pylori status and inflammation in the gastric mucosa of patients with gastric ulcer: a randomized double-blind placebo-controlled multicentre trial. Aliment Pharmacol Ther 18 Suppl 1:146-152, 2003.

30. Choe, J.-Y., Park, K.-Y., Lee, S.-J., Park, S.-H., and Kim, S.-K. Rebamipide inhibits tumor necrosis factor- $\alpha$-induced interleukin- 8 expression by suppressing the NF- $\mathrm{B}$ signal pathway in human umbilical vein endothelial cells. Inflamm Res 59:1019-1026, 2010.

31. Ogawa M, Simsek C, Kojima T, et al. The Effect of Rebamipide Ophthalmic Solution on Cytokine and Mucin Secretion in Culture of Conjunctival Epithelial Cells From the $\mathrm{Cu}, \mathrm{Zn}$-Superoxide Dismutase-1 (SOD-1) Knock-Down Mouse. Eye \& Contact Lens 2019;45:93-8.

32. Li, W., Hayashida, Y., Chen, Y.T., et al. Air exposure induced squamous metaplasia of human limbal epithelium. Invest Ophthalmol Vis Sci 49:154-162, 2008.

33. Li, W., Chen, Y.T., Hayashida, Y., et al. Down-regulation of Pax6 is associated with abnormal differentiation of 
corneal epithelial cells in severe ocular surface diseases. J Pathol 214:114-122, 2008.

34. Murube, J., and Rivas, L. Impression cytology on conjunctiva and cornea in dry eye patients establishes a correlation between squamous metaplasia and dry eye clinical severity. Eur J Ophthalmol 13:115-127, 2003.

35. Soong, H.K., Martin, N.F., Wagoner, M.D., et al. Topical retinoid therapy for squamous metaplasia of various ocular surface disorders: a multicenter, placebo-controlled doublemasked study. Ophthalmology 95:1442-1446, 1988.

36. Chen, Y.T., Li, S., Nikulina, K., Porco, T., Gallup, M., and McNamara, N. Immune profile of squamous metaplasia development in autoimmune regulator-deficient dry eye. Mol Vis 15:563-576, 2009.

37. Li, S., Gallup, M., Chen, Y.T., and McNamara, N.A. Molecular mechanism of proinflammatory cytokine-mediated squamous metaplasia in human corneal epithelial cells. Invest Ophthalmol Vis Sci 51:2466-2475, 2010.

38. Kinoshita, S., Kiorpes, T.C., Friend, J., and Thoft, R.A. Goblet cell density in ocular surface disease: a better indicator than tear mucin. Arch Ophthalmol 101:1284-1287, 1983.

39. Thoft, R.A., Friend, J., Kinoshita, S., Nikolic, L., and Foster, C.S. Ocular cicatricial pemphigoid associated with hyperproliferation of the conjunctival epithelium. Am J Ophthalmol 98:37-42, 1984.
40. Argueso, P., Balaram, M., Spurr-Michaud, S., Keutmann, H.T., Dana, M.R., and Gipson, I.K. Decreased levels of the goblet cell mucin MUC5AC in tears of patients with Sjogren syndrome. Invest Ophthalmol Vis Sci 43:10041011, 2002.

41. Rivas, L., Oroza, M.A., Perez-Esteban, A., and Murubedel-Castillo, J. Morphological changes in ocular surface in dry eyes and other disorders by impression cytology. Graefes Arch Clin Exp Ophthalmol 230:329-334, 1992.

Received: November 18, 2018

Accepted: April 26, 2019

Address correspondence to:

Dr. Jun Shimazaki

Department of Ophthalmology

Tokyo Dental College

Ichikawa General Hospital

Sugano 5-11-13, Ichikawa city

Chiba 272-8513

Japan

E-mail: jun@eyebank.or.jp 